monitoring drugs after marketing. An example of its usefulness was provided by the Boston Collaborative Drug Surveillance Program, which found that $26^{\circ}{ }_{0}$ of 105 patients given ethacrynic acid intravenously developed gastrointestinal bleeding, compared with $4.5 \%$ of other patients. ${ }^{7}$ None of the attending doctors attributed the bleeding to this drug. The limited experience of individual doctors and lack of prior suspicion prevented them from guessing the cause, but combining their observations led to discovery of an important reaction.

How can event-recording be accomplished in clinical trials? Doctors already ask patients about their symptoms. Use of checklists increases the number of symptoms elicited, but may interfere with detection of the important ones. ${ }^{8}$ Patient questionnaires may be useful. ${ }^{9}$ The only developments needed are methods for recording all adverse events and for transmitting them to the co-ordinating centre. There they must be coded and analysed. This will require extra effort and expense, but need not prolong the trial period.

Because they include a few thousand patients at most, clinical trials of new drugs can provide information about only common adverse effects. ${ }^{10}$ This has led to pessimism about the value of trials in detecting toxicity, and attention has been focused on the undeniable importance of post-marketing surveillance. There are, however, several reasons why we should make the most of the trials that are done. Firstly, the commonest reactions are often the most important. Secondly, minor complaints may be a clue to more serious (although less common) toxicity, as would have been the case with practolol. ${ }^{1}$ Thirdly, suspicions raised by clinical trials, even when firm conclusions are not possible, may lead to more effective monitoring after a drug is released. ${ }^{11}$

It has recently been suggested that all doctors using new drugs should report adverse events to the Committee on Safety of Medicines, but it is doubtful if compliance could be ensured without some provision for automatically recording the use of the drugs, as Inman has proposed. ${ }^{12}{ }^{13}$ Event-recording in clinical trials is even more urgent and would be easier to implement. Its introduction could make the initial clinical trials much more effective.

\section{References}

${ }^{1}$ Skegg, D C G, and Doll, R, Lancet, 1977, 2, 475.

2 Department of Health and Social Security, Notes on Applications for Clinical Trial Certificates. Unpublished, 1977.

${ }^{3}$ Wiseman, R A, Postgraduate Medical fournal, 1971, 47, suppl No 2, 68.

4 Vere, D W, Adverse Drug Reaction Bulletin, 1976, No 60.

${ }^{5}$ Karch, F E, et al, Clinical Pharmacology and Therapeutics, 1976, 19, 489.

${ }^{6}$ Finney, D J, Fournal of Chronic Diseases, 1965, 18, 77.

$?$ Jick, H, et al, fournal of the American Medical Association, 1970, 213, 1455.

${ }^{8}$ Huskisson, E C, and Wojtulewski, J A, British Medical Fournal, 1974, 2, 698.

9 Bulpitt, C J, and Dollery, C T, British Medical fournal, 1973, 3, 485.

10 Dollery, C T, Fournal of the Royal College of Physicians, 1977, 11, 226.

11 Anello, C, in Drug Monitoring, ed F Gross and W H W Inman. London, Academic Press, 1977.

${ }_{12}$ British Medical fournal, 1977, 1, 861.

${ }^{13}$ Inman, W H W, in Drug Monitoring, ed F Gross and W $\mathrm{H}$ W Inman. London, Academic Press, 1977.

\title{
Breast carcinoma associated with pregnancy: a clinician's dilemma
}

\author{
G G RIBEIRO, M K PALMER
}

British Medical fournal, 1977, 2, 1524-1527

\section{Introduction}

Management of breast carcinoma associated with pregnancy may be a severe test for any clinician. Widely differing views are held by surgeons, gynaecologists, and radiotherapists about its treatment and prognosis. In addition, non-clinical religious, psychological, and socioeconomic considerations will influence the choice of management.

The rarity of breast carcinoma during pregnancy means that no one institution can accumulate a wide experience, even over a long period. Only two large personal series have been published. Holleb and Farrow ${ }^{1}$ reported on 283 patients from the Memorial Hospital for Cancer and Allied Diseases and Peters ${ }^{2}$ from the Ontario Cancer Institute reported on 126 patients in 1962, extending the series to 295 patients in $1968 .^{3}$ Based on her extensive studies, Peters expressed strong views on the management of breast carcinoma associated with pregnancy. Clinicians have probably been most influenced by her views, which are widely quoted, and we have therefore compared and contrasted our findings with those of Peters.

Christie Hospital and Holt Radium Institute, Manchester M20 9BX G G RIBEIRO, MB, FRCR, consultant radiotherapist

M K PALMER, BSC, FSS, senior medical statistician

\section{Patients, methods, and analysis}

Our series consisted of 128 patients with carcinoma of the breast who were pregnant. From 1941 to 1969 all these patients were referred to the Christie Hospital and Holt Radium Institute, Manchester, where they were divided into two groups.

Concurrent pregnancy group-Patients where the breast carcinoma was diagnosed during pregnancy or up to one year after childbirth.

Subsequent pregnancy group-Patients who became pregnant after completing treatment for primary breast carcinoma.

Eighty-eight patients were studied in the concurrent group and 40 patients in the subsequent pregnancy group. Three patients had two pregnancies each and therefore were included in both groups.

We have preferred to divide the phases of pregnancy into trimesters rather than into two halves as Peters has done, as we think that the former is more relevant to the practice of gynaecology and obstetrics in Britain.

Survival curves for various groups were calculated by the life-table method and compared by using the log-rank test ${ }^{4} ;$ this test was chosen because it compares the entire distributions of survival times rather than just the percentages of survivors at a fixed point in time-for example, five years.

Staging-All the patients had histological verification of carcinoma. The pathological node state was also known in all the operable patients and was taken into account when staging the disease. Staging was according to the International Clinical Staging."

Incidence-T $\mathrm{T}$ White ${ }^{6}$ reviewed 1375 cases of breast carcinoma associated with pregnancy reported from 1866 to 1953 and added 38 cases to extend the series to 1413 patients. ${ }^{7} \mathrm{He}$ calculated the frequency as three cases of breast carcinoma per 10000 pregnancies, or $2.8 \%$ of all breast carcinomas. Peters ${ }^{3}$ placed the figure slightly higher at $3.8 \%$ of all breast carcinomas. Almost 31000 cases of breast carcinoma 
were seen at the Christie Hospital from 1941 to 1969, and only 128 $(0.4 \%)$ were associated with pregnancy. About 5400 patients were aged 45 years and under, and the percentage associated with pregnancy was then $2 \cdot 8$. The low percentage at the Christie Hospital was due to the policy of giving women a prophylactic castration as part of their initial curative treatment for breast carcinoma.

\section{Results}

CONCURRENT PREGNANCY GROUP

Age

Twenty-one patients in this group of 88 were aged 40 and over at the time of becoming pregnant; the youngest was 21 and the oldest 47 .

\section{Symptoms and signs}

Most patients complained of a lump in the breast. Two patients had difficulty with breast-feeding, and two patients had reddening of the skin of the breast, which was thought to be caused by a breast abscess. The mean duration of signs and symptoms was 10 months. Fourteen patients were aware of a lump in the breast from two months to 13 years before pregnancy and took action only because they thought the size of the lump had increased during pregnancy. The remaining patients became aware of the lump during pregnancy.

\section{Primary treatment of the breast carcinoma}

Sixty-nine of the 88 patients had operable tumours, and, of these, 28 had a radical mastectomy and 34 a radical mastectomy followed by radical radiotherapy. The remaining seven patients had a local mastectomy with axillary node dissection followed by radical radiotherapy. Ten patients were inoperable and had only radical radiotherapy. Eight patients had palliative radiotherapy, and one patient died before treatment could start.

Of the 69 operable patients, $89 \%$ were found to have positive nodes in the ipsilateral axilla; this percentage is considerably higher than the $74 \%$ reported by Montgomery ${ }^{8}$ and the $72 \%$ reported by Holleb and Farrow. ${ }^{1}$

\section{Survival}

The total five-year survival of $34 \%$ (table I) was very close to the $33 \%$ reported by Peters." The worsening survival from stage I to stage IV was statistically highly significant $(P<0.001)$. We are unable to provide statistics for the survival of the total premenopausal series from 1941 to 1969 , but Peters ${ }^{3}$ did find that stage for stage, the fiveyear survival rate of the concurrent group was 10 to $15 \%$ lower than in the total premenopausal series.

TABLE I-Percentage of survivors at 5 and 10 years of 88 patients in concurrent pregnancy group by clinical stage

\begin{tabular}{c|c|c|c}
\hline \multirow{2}{*}{ Clinical stage } & No of patients & \multicolumn{2}{|c}{${ }^{\circ}$ "s Survivors } \\
\cline { 2 - 3 } & & 5 years & 10 years \\
\hline II & 10 & 90 & 90 \\
III & 49 & 37 & 21 \\
IV & 20 & 15 & 0 \\
\hline Total & 88 & 0 & 24 \\
\hline
\end{tabular}

We found from analysis according to the phase of pregnancy during which the breast carcinoma was discovered (fig 1) that the best survival rate was in the group who discovered their disease in the third trimester; the overall difference between the six groups is not, however, statistically significant $(P=0 \cdot 84)$. The findings were similar when only the data on stage I and stage II cases were analysed.

One of the most striking findings of Peters, ${ }^{2} 3$ however, was the very poor survival of patients treated in the second half of pregnancy compared with those treated in the first half or the immediate postpartum period. White ${ }^{6}$ had previously noted this but thought that the reason was the greater number of patients with advanced disease presenting in the second half of pregnancy. Peters ${ }^{3}$ found no excess

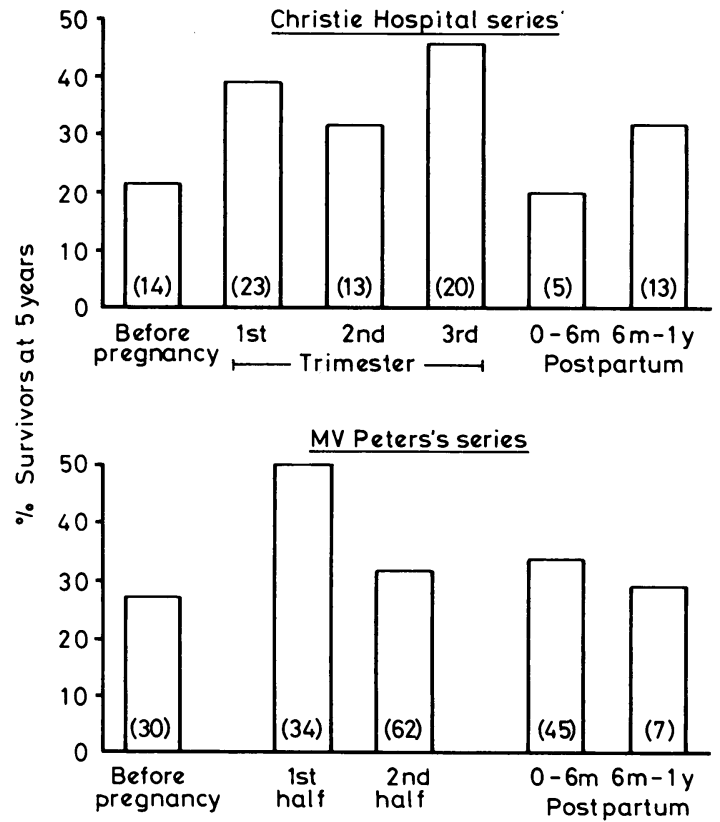

FIG 1-Five-year survival in concurrent pregnancy group related to phase of pregnancy at which carcinoma was discovered.

of advanced cases, and her findings were the same when only stage I and stage II cases were analysed.

We found that, while the patients treated in the first trimester had the highest percentage of five-year survivors (fig 2), the difference in survival between the five groups was not statistically significant $(P=0.81)$. The findings were the same when only stage I and stage II cases were analysed.

In our series 24 patients with operable breast carcinoma were treated in the second and third trimesters: 14 of the 24 patients had presented in the same phase of pregnancy and seven survived more than five years. Ten of the 24 patients were not treated until the early postpartum phase and five of them survived more than five years. From this evidence there would appear to be no special risk attached to curative treatment being carried out for operable breast carcinoma in the latter half of pregnancy.

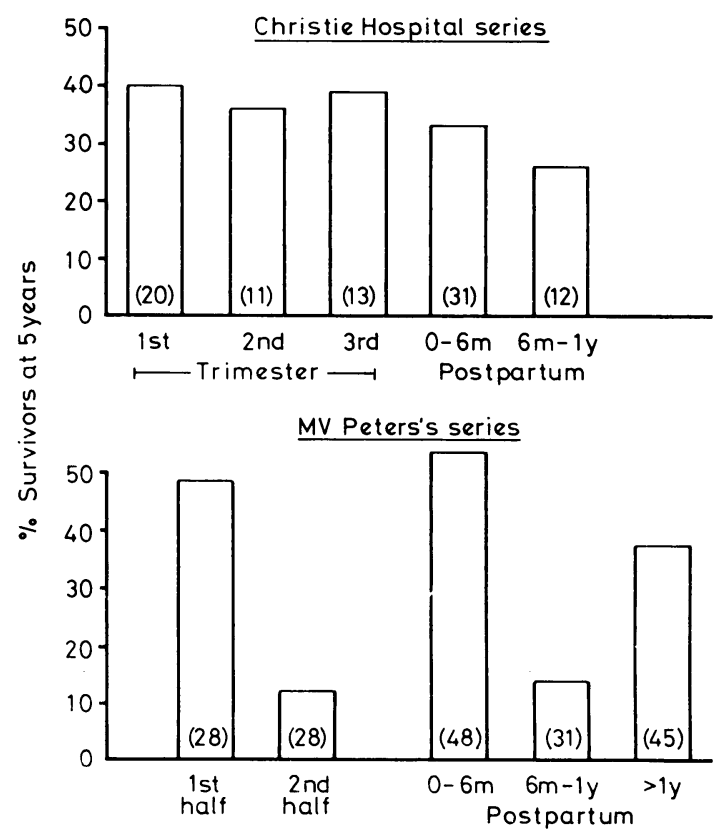

FIG 2-Five-year survival in concurrent pregnancy group related to phase of pregnancy at which patient was treated. 


\section{Outcome of pregnancy}

Seventy-three of the 88 pregnancies ended in the delivery of a normal live child. There were two stillbirths and one spontaneous abortion. Twelve patients had their pregnancy terminated, two in the first trimester and one in the second trimester. Ten of these twelve patients had advanced disease and died in less than five years, but two are alive and well at 15 and 31 years.

\section{SUBSEQUENT PREGNANCY GROUP}

Only 40 patients became pregnant after definitive treatment for their primary breast carcinoma: the youngest patient was 23 and the oldest 44.

\section{Treatment}

All 40 patients had breast tumours considered to be operable: 23 patients had only a radical mastectomy, 16 patients had a radical mastectomy plus radiotherapy, and one patient had a local mastectomy plus radiotherapy. Of the 40 patients, 25 had positive nodes in the ipsilateral axilla on histological examination.

\section{Survival}

The overall survival of $70 \%$ at five years (table II) compared favourably with the $75 \%$ reported by Peters $^{3}$ in her series of 87 patients. The trend for worsening survival with increasing stage of the disease was statistically highly significant $(P=0.003)$.

The survival curve of the total group of 40 patients was compared with the survival curve of 120 controls matched for age, stage, year of treatment, and disease-free interval. The matched control had to have survived for at least the length of time that had elapsed between the other patient's treatment and her pregnancy. Thus the only

TABLE II-Percentage of survivors at 5 and 10 years of 40 patients in subsequent pregnancy group by clinical stage

\begin{tabular}{c|c|c|c}
\hline \multirow{2}{*}{ Clinical stage } & No of patients & \multicolumn{2}{|c}{ ", Survivors } \\
\cline { 2 - 3 } & & 5 years & 10 years \\
\hline I & 15 & 87 & 79 \\
II & 6 & 58 & 52 \\
\hline Total & 40 & 60 & 20 \\
\hline & 60 & 59 \\
\hline
\end{tabular}

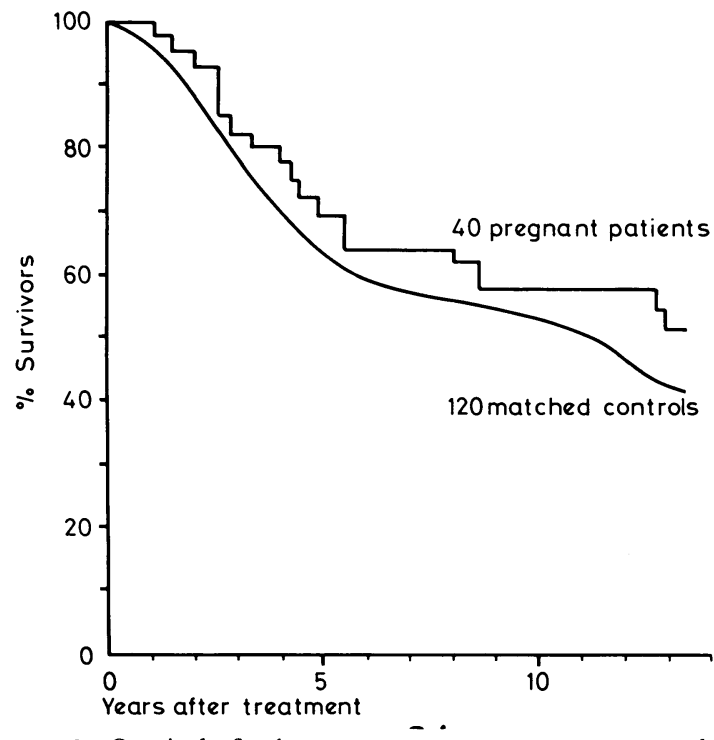

FIG 3-Survival of subsequent pregnancy group compared with that of matched controls.

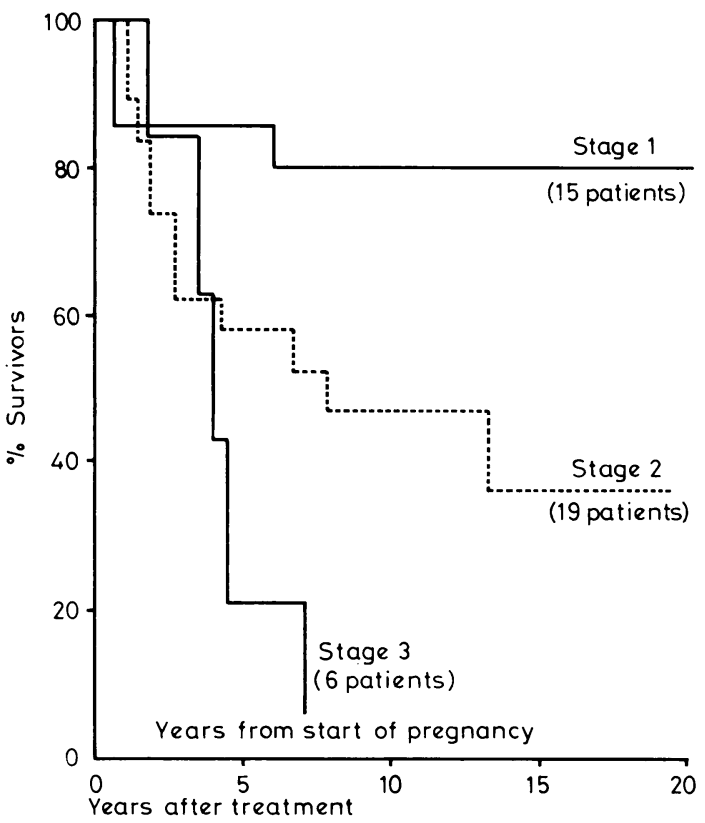

FIG 4-Survival by stage of subsequent pregnancy group measured from start of pregnancy.

difference between any two matched patients would be the pregnancy. The results of the comparison (fig 3) show that while the survival of the subsequent pregnancy group was slightly better than that of the controls, the difference was not statistically significant $(P=0.40)$.

Survival for the subsequent pregnancy group was also related to the interval between mastectomy and pregnancy. Peters ${ }^{3}$ found that $54 \%$ of patients survived five years even when pregnancy occurred within six months of mastectomy. Our findings substantiate this fact, and $69 \%$ of all patients survived five years. More important and useful information may be obtained by measuring survival from the month pregnancy started for each stage of breast carcinoma (fig 4). There was an obvious worsening of survival with increasing stage of disease that was statistically highly significant $(P=0.0005)$. While $69 \%$ of the whole group survived five years from the start of pregnancy, half of the stage II patients and all of the stage III patients were dead within seven years.

\section{Conclusions}

We think the following conclusions on the management of breast carcinoma associated with pregnancy may reasonably be drawn from these findings.

Patients presenting with early breast carcinoma in the first trimester should be treated in the same manner as non-pregnant patients. There is no harm in allowing the pregnancy to continue, unless it is against the express views of the patient and her husband. In several centres, however, patients with operable tumours and positive nodes are being entered into adjuvant chemotherapy trials. If it ultimately transpires that more women will be cured of breast carcinoma by cytotoxic drugs then there may be a new argument for aborting the fetus if the disease is treated in the first trimester because of the teratogenic risks of these drugs.

In this series we could find no special risk in treating patients with breast carcinoma in the second half of pregnancy rather than waiting for the postpartum phase. It would be unreasonable for a patient who knew the diagnosis to be told that no treatment was to take place for some weeks. Equally, perhaps not all surgeons would stand by and do nothing.

For patients with an early breast carcinoma definitive surgery may be performed if the delivery is still some weeks away and the pregnancy may be allowed to continue; while closer to the delivery date a simple mastectomy could be followed by radiotherapy after the birth. Patients with stage III and stage IV 
disease have such a poor prognosis that the pregnancy should be terminated and appropriate treatment started.

Patients found to have a breast carcinoma during lactation should have lactation suppressed by means other than stilboestrol and the carcinoma treated in the usual way.

While the overall survival of the subsequent pregnancy group was not significantly worse than that of matched controls, half of the stage II and all of the stage III patients were dead within seven years of the start of pregnancy.

Ultimately, despite the results and conclusions of this and other reports, treatment in this very complex situation will have to be tailored to the individual patient. What is considered to be the best treatment for the patient by the clinicians may well not be accepted by the patient, and her wishes should not be ignored.

We thank Dr M V Peters and E and S Livingstone Ltd for giving permission to reproduce the findings of Dr Peters (figs 2 and 3). We also thank Miss Sheila Hull for help with the statistical analysis, Dr M P Cole for helpful advice, the medical illustration department, and Mrs Hurst for typing the manuscript.

\section{References}

${ }^{1}$ Holleb, A I, and Farrow, J H, Surgery, Gynecology and Obstetrics, 1962, $115,65$.

${ }^{2}$ Peters, M V, Radiology, 1962, 78, 58.

${ }^{3}$ Peters, M V, Prognostic Factors in Breast Cancer, p 65. London \& Edinburgh, E and S Livingstone Ltd, 1968.

1 Peto, R, et al, British fournal of Cancer, 1977, 35, 1 .

Classification of Malignant Tumours. Geneva, UICC, 1968.

${ }^{6}$ White, T T, American fournal of Obstetrics and Gynecology, 1955, 69, 1277.

- White, T T, Surgery, Gynecology and Obstetrics, 1955, 100, 661.

Montgomery, T L, American fournal of Obstetrics and Gynecology, 1961, $81,926$.

(Accepted 20 October 1977)

\title{
Defective blood glucose counter-regulation in diabetics is a selective form of autonomic neuropathy
}

\author{
L V CAMPBELL, E W KRAEGEN, L LAZARUS
}

\section{Background}

Some data on the mechanism of glucoregulation in hypoglycaemia have been gained from using the insulin tolerance test. This test $(0 \cdot 1-0 \cdot 6 \mathrm{U} / \mathrm{kg}$ neutral insulin by intravenous bolus) produces a rapid, inevitable hypoglycaemia in both normal subjects and diabetics. This evokes a standard stress response with release of catecholamines, glucagon, cortisol, growth hormone, and prolactin. ${ }^{1}$ The release of growth hormone and cortisol is not essential in immediate restoration of blood glucose after hypoglycaemia, ${ }^{2}$ although chronic deficiency of either can cause hypoglycaemia. ${ }^{3}$ In patients under sympathetic blockade, ${ }^{4}$ with cervical cord lesions, ${ }^{5}$ and after adrenalectomy ${ }^{6}$ the sympathoadrenal system does not affect blood glucose restoration during insulin hypoglycaemia. In these patients the glucagon response was also unaffected by the sympathoadrenal system.

Other conflicting data suggest that catecholamines do contribute to restoration of blood glucose during hypoglycaemia, ${ }^{7}$ 8 although their major role probably lies in mobilising alternate substrate for energy production. ${ }^{9}$ In mild hypoglycaemia, however, the blood glucose concentration is restored without adrenaline release. ${ }^{10}$ Thus the release of glucagon is the most essential means of hypoglycaemia counter-regulation. There is evidence from studies of the effects of vagotomy ${ }^{11}$ and atropine $^{12}{ }^{13}$ that an intact vagus is necessary for release of glucagon to offset impending hypoglycaemia. This is consistent with the reports of parasympathetic innervation of the $\alpha$-cell ${ }^{14}{ }^{15}$ and of the efficacy of acetylcholine in the direct stimulation of glucagon release. ${ }^{16}{ }^{17}$ A low blood glucose has been shown to be a direct stimulus to the $\alpha$-cell in vitro when the glucose concentration in the medium is very low ${ }^{18}{ }^{19}$; this response can be suppressed by the presence of non-esterified fatty acid concentrations a 